Supporting information

\title{
Prussian Blue: a Potential Material to Improve the Electrochemical Performance of Lithium-Sulfur Batteries
}

Yueying Peng ${ }^{\mathrm{a}}$, Bing $\mathrm{Li}^{\mathrm{b}}$, Yunhui Wang ${ }^{\mathrm{a}}$, Xinyi $\mathrm{He}^{\mathrm{a}}$, Jianxing Huang ${ }^{\mathrm{a}}$, and Jinbao Zhao ${ }^{* a, b}$

${ }^{a}$ State Key Lab of Physical Chemistry of Solid Surfaces, Collaborative Innovation Centre of Chemistry for Energy Materials, College of Chemistry and Chemical Engineering, Xiamen University, Xiamen, 361005, P.R. China, Email: Jbzhao@,xmu.edu.cn.

${ }^{\mathrm{b}}$ College of Energy, Xiamen University, Xiamen, 361005, P.R. China. 


\section{Figures and tables}

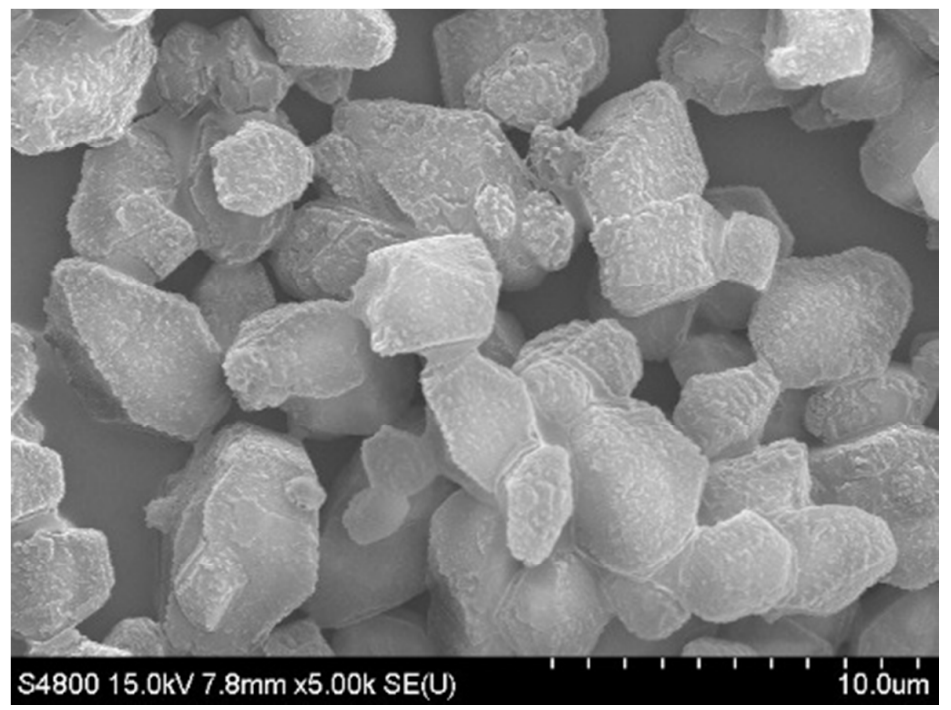

Fig. S1 SEM images of commercial sulfur powders.

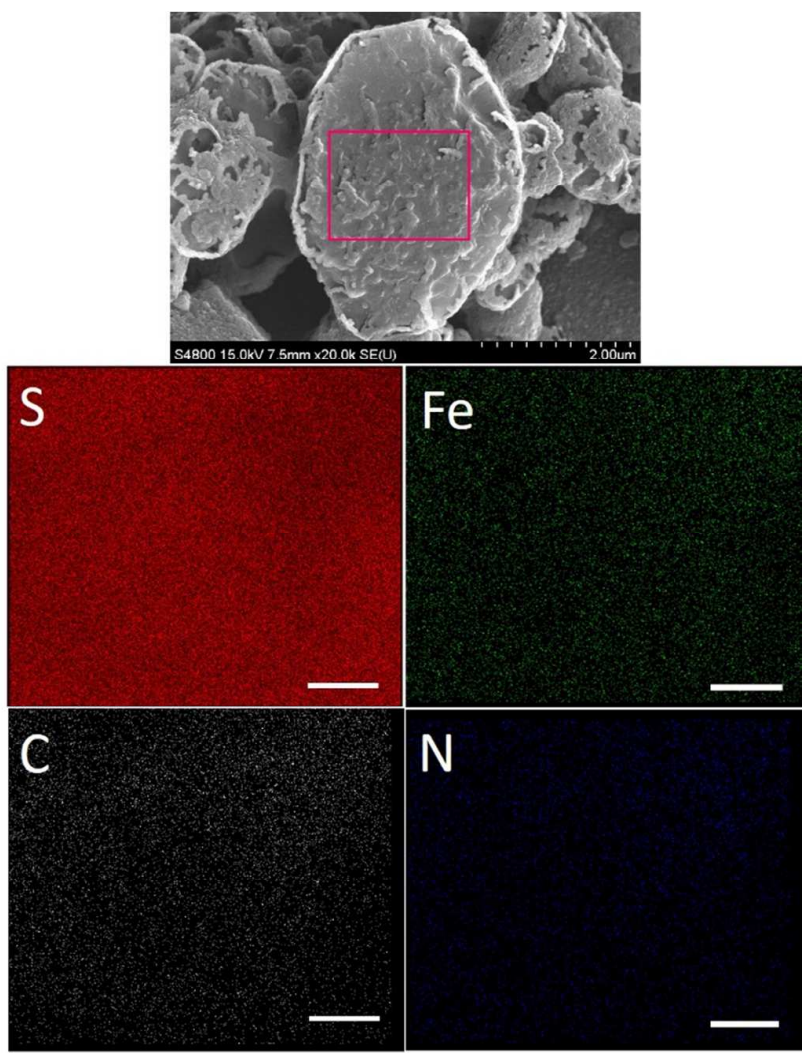

Fig. S2 SEM image of 10SPB and corresponding elemental mapping of S, Fe, C, and $\mathrm{N}($ bar $=200 \mathrm{~nm})$. 


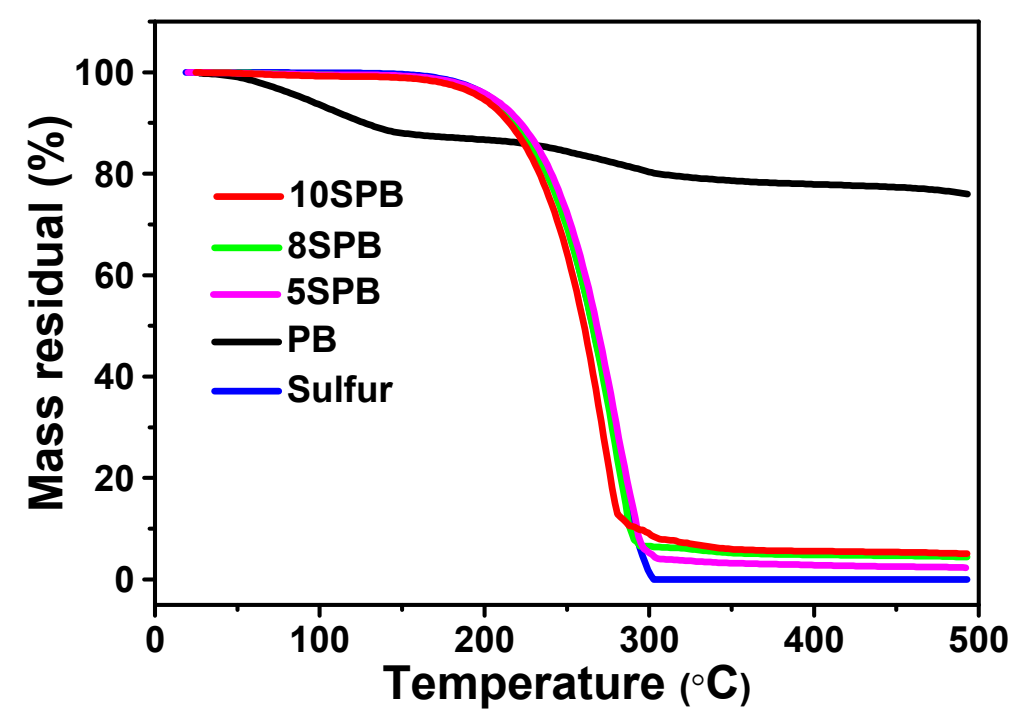

Fig. S3 TGA curves of sufur, PB and SPB composites in $\mathrm{N}_{2}$

Table S1 Weight percentage according to TGA curves

\begin{tabular}{llll}
\hline Weight percentage & $5 \mathrm{SPB}$ & $8 \mathrm{~PB}$ & $10 \mathrm{SPB}$ \\
\hline $\mathrm{T}=306^{\circ} \mathrm{C}$ & $4.0 \%$ & $6.4 \%$ & $7.9 \%$ \\
$\mathrm{~PB}$ & $5.0 \%$ & $8.0 \%$ & $9.9 \%$ \\
Sulfur & $95.0 \%$ & $92.0 \%$ & $90.1 \%$ \\
\hline
\end{tabular}



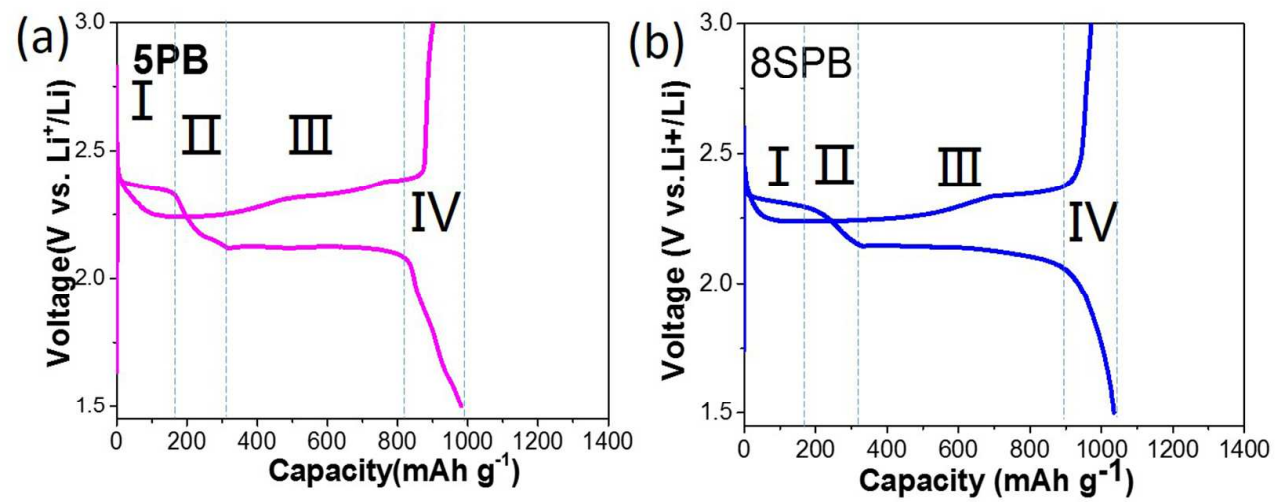

Fig. S4 Initial discharge/charge profiles for Li-S cells at $0.2 \mathrm{~A} \mathrm{~g}^{-1}$ : (a) 5SPB, (b) 8SPB.

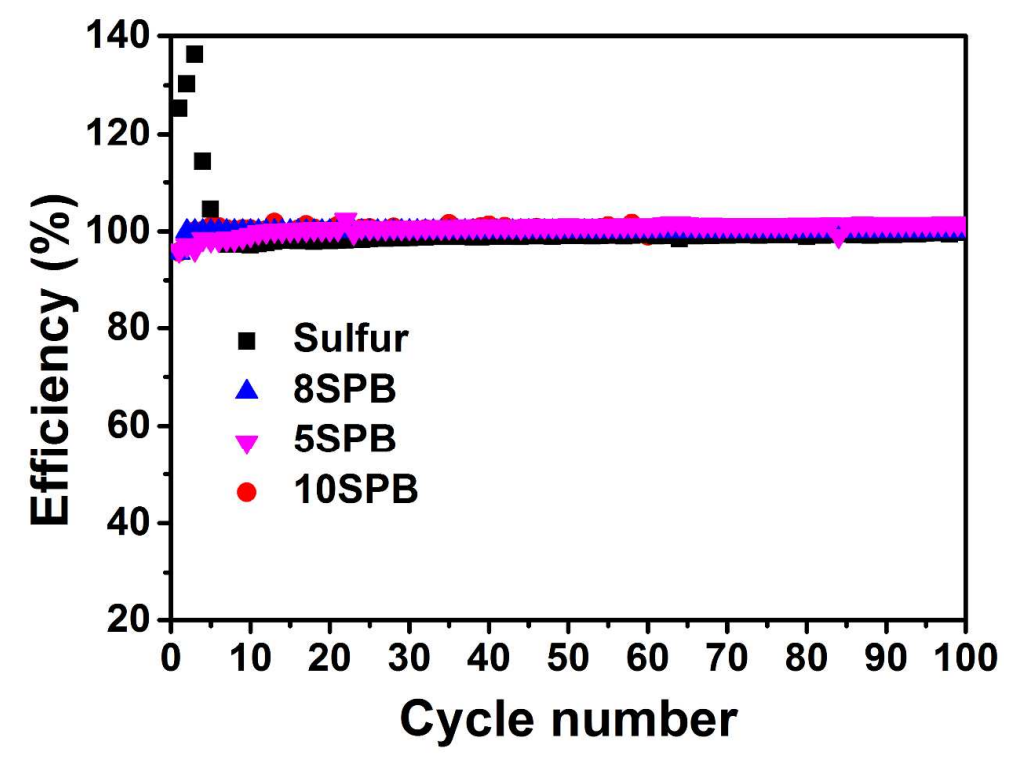

Fig. S5 The efficiency of sulfur, 5SPB, 8SPB and 10SPB cathodes at $0.2 \mathrm{~A} \mathrm{~g}^{-1}$ over 100 cycles. 
Table S2 Cycling performance of various sulfur cathode materials

\begin{tabular}{llllllll}
\hline Materials & $\begin{array}{l}\text { Sulfur } \\
\text { loading }\end{array}$ & Rate $^{\mathrm{b}}$ & Cycles & $\begin{array}{l}\text { Initial } \\
\text { discharge } \\
\text { capacity } \\
\left(\mathrm{mAh} \mathrm{g}^{-1}\right)\end{array}$ & $\begin{array}{l}\text { Retained } \\
\text { capacity } \\
(\mathrm{mAh}\end{array}$ & $\begin{array}{l}\text { Capacity } \\
\text { retention }\end{array}$ & Ref. \\
& & & & & & \\
\hline$\gamma-\mathrm{Al}_{2} \mathrm{O}_{3} / \mathrm{S}$ & $50 \%$ & $0.06 \mathrm{C}$ & 25 & 750 & 660 & $88 \%$ & 1 \\
$\mathrm{Fe}_{2} \mathrm{O}_{3} / \mathrm{S}$ & $66.5 \%$ & $0.5 \mathrm{C}$ & 30 & 544.5 & 422 & $77.5 \%$ & 2 \\
$\mathrm{hollowSnO}_{2} / \mathrm{S}$ & $46 \%$ & $0.5 \mathrm{C}$ & 50 & 1176 & 736.6 & $62.6 \%$ & 3 \\
$\mathrm{MgO}^{-\mathrm{S}}$ & $54 \%$ & $0.2 \mathrm{C}$ & 100 & $\sim 866^{\mathrm{c}}$ & $\sim 725^{\mathrm{c}}$ & $83.8 \%$ & 4 \\
$\mathrm{TiS}_{2} / \mathrm{S}$ & $48 \%$ & $0.1 \mathrm{C}$ & 100 & 1020 & $\sim 720^{\mathrm{c}}$ & $70.6 \%$ & 5 \\
$\mathrm{MoS}_{2} / \mathrm{S}$ & $37.5 \%$ & $0.2 \mathrm{C}$ & 1000 & $\sim 1275^{\mathrm{c}}$ & $\sim 300^{\mathrm{c}}$ & $23.5 \%$ & 6 \\
$\mathrm{FeS}_{2} / \mathrm{S}$ & $67 \%$ & $0.5 \mathrm{mAcm}{ }^{-2}$ & 100 & $\sim 1260^{\mathrm{c}}$ & 700 & $55.5 \%$ & 7 \\
$\mathrm{~PB} / \mathrm{S}$ & $63 \%$ & $0.12 \mathrm{C}$ & 50 & 1112 & 825 & $74.2 \%$ & this \\
& & & 100 & 1112 & 722 & $64.9 \%$ & work \\
\hline
\end{tabular}

a. the mass percentage of sulfur on the entire cathode

b. $1 \mathrm{C}=1678 \mathrm{~mA} \mathrm{~g}^{-1}$

c. the capacity is calculated from the figures as the authors did not provide the specific number

\section{References:}

1. Choi, Y. J.; Jung, B. S.; Lee, D. J.; Jeong, J. H.; Kim, K. W.; Ahn, H. J.; Cho, K. $\mathrm{K}$; $\mathrm{Gu}, \mathrm{H} . \mathrm{B}$., Electrochemical properties of sulfur electrode containing nano Al2O3 for lithium/sulfur cell. Phys. Scr. 2007, T129, 62-65.

2. Zhao, C.; Shen, C.; Xin, F.; Sun, Z.; Han, W., Prussian blue-derived Fe2O3/sulfur composite cathode for lithium-sulfur batteries. Mater. Lett. 2014, 137, 52-55.

3. Zhang, L. P.; Wang, Y. F.; Gou, S. Q.; Zeng, J. H., All Inorganic Frameworks of Tin Dioxide Shell as Cathode Material for Lithium Sulfur Batteries with Improved Cycle Performance. J. Phys. Chem. C 2015, 119 (52), 28721-28727.

4. Ponraj, R.; Kannan, A. G.; Ahn, J. H.; Kim, D. W., Improvement of Cycling Performance of Lithium-Sulfur Batteries by Using Magnesium Oxide as a Functional Additive for Trapping Lithium Polysulfide. ACS Appl. Mater. Interfaces 2016, 8 (6), 4000-4006. 
5. Garsuch, A.; Herzog, S.; Montag, L.; Krebs, A.; Leitner, K., Performance of Blended TiS2/Sulfur/Carbon Cathodes in Lithium-Sulfur Cells. ECS Electrochem. Lett. 2012, 1 (1), A24-A26.

6. Dirlam, P. T.; Park, J.; Simmonds, A. G.; Domanik, K.; Arrington, C. B.; Schaefer, J. L.; Oleshko, V. P.; Kleine, T. S.; Char, K.; Glass, R. S.; Soles, C. L.; Kim, C.; Pinna, N.; Sung, Y. E.; Pyun, J., Elemental Sulfur and Molybdenum Disulfide Composites for Li-S Batteries with Long Cycle Life and High-Rate Capability. ACS Appl. Mater. Interfaces 2016, 8 (21), 13437-13448.

7. Zhang, S. S.; Tran, D. T., Pyrite FeS2as an efficient adsorbent of lithium polysulphide for improved lithium-sulphur batteries. J. Mater. Chem. A 2016, 4 (12), 4371-4374. 